\title{
A CLOSED HYPERSURFACE WITH CONSTANT SCALAR AND MEAN CURVATURES IN $\mathbb{S}^{4}$ IS ISOPARAMETRIC
}

\author{
SHAOPING CHANG
}

\section{INTRODUCTION}

A hypersurface $M^{n}$ in the unit round sphere $\mathbb{S}^{n+1}$ is called isoparametric if it has constant principal curvatures. When $n=3$, due to the work of Elie Cartan in 1939 [C], it is known that an isoparametric hypersurface $M^{3}$ is a piece of either a 3-sphere, a product of spheres, or a tube of constant radius over the Veronese embedding. It must be interesting to characterize such a hypersurface only in terms of its scalar curvature and mean curvature.

Denote by $\mathcal{H}^{n}$ the class of closed n-manifolds of constant scalar curvatures immersed in $\mathbb{S}^{n+1}$ with constant mean curvatures. One may ask the following:

QUESTION 0.1. When $n=3$, does every $M^{3} \in \mathcal{H}^{3}$ need to be isoparametric?

When either $R \geq 0$ or $H=0$, the answer has been known to be affirmative by the works of [DB] and [S, CDK, L, PT2, Ch], respectively.

In the present paper, we will demonstrate that such a conclusion is indeed valid without those further assumptions. Namely,

Classification Theorem. A closed 3-manifold of constant scalar curvature immersed in $\mathbb{S}^{4}$ is isoparametric provided it has constant mean curvature.

Our approach is to show that the technical condition $R \geq 0$ in [DB] is automatically satisfied by any compact $M^{3}$ with constant $R$ and $H$. Namely,

Main Theorem. Suppose that $M^{3}$ is a closed hypersurface of constant scalar curvature $R$ in $\mathbb{S}^{4}$ with constant mean curvature $H$. Then $R \geq 0$. 
Remark 0.1. It is believed that the sub-class $\mathcal{M}^{n}$ (of $\mathcal{H}^{n}$ ) consisting of those minimal hypersurfaces (i.e. $H=0$ ) would be quite special. Chern [Y] proposed that in every dimension $n$, all the possible values $R$ form a discrete subset of the real numbers $R$. And R. Bryant [B] suggested that in dimension 3, a piece of minimal hypersurface of constant scalar curvature has to be isoparametric.

The paper is divided into four sections. We will first present in Section 1 some terminology of theory of submanifolds, and in Section 2 a general study on our setting. The proof of the Main Theorem is completed in Section 3 and Section 4.

We will always use $i, j, k, \ldots$ for indices running over $\{1,2,3\}$ while $A, B$, $C, \ldots$ over $\{1,2,3,4\}$, and $\delta_{A B}$ to denote the Kronecker's symbol.

Acknowledgement. The author wishes to thank Prof. S.Y. Cheng for his continuous advices, supports and encouragement.

\section{Terminology and Notation}

Let $M^{3}$ be a manifold of dimension 3 immersed in a Riemannian manifold $N^{4}$ of dimension 4.

Choose a local orthonormal frame field $\left\{e_{A}\right\}$ in $N^{4}$ such that, after restricted to $M^{3}$, the $e_{j}$ 's are tangent to $M^{3}$.

Denote by $\left\{\omega_{A}\right\}$ the coframe dual to $\left\{e_{A}\right\}$ and $\left\{\omega_{A B}\right\}$ the connection forms of $N^{4}$. Then the structure equations of $N^{4}$ are given by

$$
\begin{gathered}
d \omega_{A}=\sum_{B} \omega_{A B} \wedge \omega_{B} \\
\omega_{A B}+\omega_{B A}=0 \\
d \omega_{A B}=\sum_{C} \omega_{A C} \wedge \omega_{C B}-\frac{1}{2} \sum_{C, D} K_{A B C D} \omega_{C} \wedge \omega_{D} \\
K_{A B C D}+K_{A B D C}=0
\end{gathered}
$$

We call $K_{A B C D}$, its contractions $K_{A C}=\sum_{B} K_{A B C B}$ and $K=\sum_{A, B} K_{A B A B}$, respectively, the curvature tensor, the Ricci curvature tensor and the scalar curvature of $N^{4}$, respectively.

When $N^{4}$ is the unit sphere $\mathbb{S}^{4}$, it turns out that $K_{A B C D}=\delta_{A C} \delta_{B D}-\delta_{A D} \delta_{B C}$.

Next, we restrict all tensors to $M^{3}$. 
First of all, we have $\omega_{4}=0$, and then $\sum_{i} \omega_{4 i} \wedge \omega_{i}=d \omega_{4}=0$ on $M^{3}$. By Cartan's lemma, we can write

$$
\omega_{4 i}=\sum_{j} h_{i j} \omega_{j}, \quad \text { with } \quad h_{i j}=h_{j i} .
$$

We call $h=\sum_{i, j} h_{i j} \omega_{i} \omega_{j}$, the eigenvalues $\lambda_{i}$ of matrix $\left(h_{i j}\right)$, and $H=$ $\sum_{i} h_{i i}=\sum_{i} \lambda_{i}$ the second fundamental form, the principal curvatures, and the mean curvature of $M^{3}$, respectively.

Secondly, from

$$
\begin{gathered}
d \omega_{i}=\sum_{j} \omega_{i j} \wedge \omega_{j}, \quad \omega_{i j}+\omega_{j i}=0 \\
d \omega_{i j}=\sum_{k} \omega_{i k} \wedge \omega_{k j}-\frac{1}{2} \sum_{k, l} R_{i j k l} \omega_{k} \wedge \omega_{l}
\end{gathered}
$$

we find the curvature tensor, the Ricci curvature tensor and the scalar curvature of $M^{3}$ is respectively given by

$$
\begin{aligned}
R_{i j k l} & =K_{i j k l}+h_{i k} h_{j l}-h_{i l} h_{j k} \\
R_{i k} & =2 \delta_{i k}+H h_{i k}-\sum_{j} h_{i j} h_{j k} \\
R & =6+H^{2}-\sum_{i, j} h_{i j}^{2}
\end{aligned}
$$

Note that from (1.3) the square norm $S=\sum_{i, j} h_{i j}^{2}$ of $h$ is a constant if so are both $R$ and $H$.

Given a symmetric 2-tensor $T=\sum_{i, j} T_{i j} \omega_{i} \omega_{j}$ on $M^{3}$, we also define its covariant derivatives, denoted by $\nabla T, \nabla^{2} T$ and $\nabla^{3} T$, etc. with components $T_{i j, k}, T_{i j, k l}$ and $T_{i j, k l p}$, respectively, as following:

$$
\begin{aligned}
\sum_{k} T_{i j, k} \omega_{k} & =d T_{i j}+\sum_{s}\left(T_{s j} \omega_{s i}+T_{i s} \omega_{s j}\right) \\
\sum_{l} T_{i j, k l} \omega_{l} & =d T_{i j, k}+\sum_{s}\left(T_{s j, k} \omega_{s i}+T_{i s, k} \omega_{s j}+T_{i j, s} \omega_{s k}\right) \\
\sum_{p} T_{i j, k l p} \omega_{p} & =d T_{i j, k l}+\sum_{s}\left(T_{s j, k l} \omega_{s i}+T_{i s, k l} \omega_{s j}+T_{i j, s l} \omega_{s k}+T_{i j, k s} \omega_{s l}\right) \\
& \text { etc. }
\end{aligned}
$$

We will sometimes use $\nabla_{e_{k}} T_{i j}$ to denote $T_{i j, k}$, etc.. 
EXAMPLE 1.1. $T=\sum_{i} \omega_{i}^{2}$, i.e. $T_{i j}=\delta_{i j}$.

Since $d \delta_{i j}=0$ and $\sum_{s}\left(\delta_{s j} \omega_{s i}+\delta_{i s} \omega_{s j}\right)=\omega_{j i}+\omega_{i j}=0$,

$$
\nabla T=0, \quad \text { i.e. } \quad \delta_{i j, k}=0, \quad \forall i, j, k .
$$

In general, the resulting tensors are no longer symmetric, and the rules to switch sub-indices obey to the Ricci formulas as follows:

$$
\begin{aligned}
T_{i j, k l}-T_{i j, l k} & =\sum_{s}\left(T_{s j} R_{s i k l}+T_{i s} R_{s j k l}\right) \\
T_{i j, k l p}-T_{i j, k p l} & =\sum_{s}\left(T_{s j, k} R_{s i l p}+T_{i s, k} R_{s j l p}+T_{i j, s} R_{s k l p}\right) \\
T_{i j, k l p m}-T_{i j, k l m p} & =\sum_{s}\left(T_{s j, k l} R_{s i p m}+T_{i s, k l} R_{s j p m}+T_{i j, s l} R_{s k p m}+T_{i j, k s} R_{s l p m}\right) \\
\text { etc. } &
\end{aligned}
$$

EXAMPLE 1.2. $T=h=\sum_{i, j} h_{i j} \omega_{i} \omega_{j}$ with $N^{4}=\mathbb{S}^{4}$.

For the sake of simplicity, we always omit the comma (,) between indices in this special case.

Recall that

$$
\begin{gathered}
\omega_{4 i}=\sum_{j} h_{i j} \omega_{j}, \\
d \omega_{4 i}=\sum_{C} \omega_{4 C} \wedge \omega_{C i}-\frac{1}{2} \sum_{C, D} K_{4 i C D} \omega_{C} \wedge \omega_{D}
\end{gathered}
$$

Since $K_{A B C D}=\delta_{A C} \delta_{B D}-\delta_{A D} \delta_{B C}$ for $\mathbb{S}^{4}$ and $\omega_{4}=0$ on $M^{3}$, the second term on the right hand side of (1.5) vanishes on $M^{3}$.

By differentiating (1.4) and applying both (1.5) and (1.4) to the resulting equation, we find

$$
d\left(\sum_{j} h_{i j} \omega_{j}\right)=\sum_{j, k} h_{j k} \omega_{k} \wedge \omega_{j i}
$$

It follows that $\forall i$,

$$
\sum_{j}\left[d h_{i j}+\sum_{k}\left(h_{k j} \omega_{k i}+h_{i k} \omega_{k j}\right)\right] \wedge \omega_{j}=0
$$


i.e.

$$
\sum_{j, k} h_{i j k} \omega_{k} \wedge \omega_{j}=0
$$

Therefore, $h_{i j k}$ is symmetric in all the indices.

Moreover, in the event of $H=$ constant, for $\forall i, j$,

$$
\begin{aligned}
\sum_{k} h_{i j k k}= & \sum_{k} h_{k i j k}=\sum_{k}\left[h_{k i k j}+\sum_{m}\left(h_{m i} R_{m k j k}+h_{k m} R_{m i j k}\right)\right] \\
= & \sum_{m} h_{m i}\left(2 \delta_{m j}+H h_{m j}-\sum_{k} h_{m k} h_{k j}\right) \\
& +\sum_{k, m} h_{k m}\left(\delta_{m j} \delta_{i k}-\delta_{m k} \delta_{i j}+h_{m j} h_{i k}-h_{m k} h_{i j}\right) \\
= & 3 h_{i j}+H \sum_{m} h_{m i} h_{m j}-H \delta_{i j}-S h_{i j}
\end{aligned}
$$

It follows that (cf. [CY])

$$
\frac{1}{2} \Delta S=\sum_{i, j, k} h_{i j k}^{2}+(3-S) S-H^{2}+H f_{3}
$$

here and afterforth, for each $k \geq 3, f_{k}=\sum_{i} \lambda_{i}^{k}$.

We finish the current section by noting the following combination formula

$$
\sum_{i, j, k}=6 \sum_{i, j, k \text { distinct }}+\sum_{i=j=k}+3 \sum_{i=j \neq k}
$$

as long as the summand is symmetric in the indices $i, j$, and $k$. It will be applied whenever we want to compute a summation explicitly.

\section{A General Discussion when both $R$ and $H$ are Constant}

From now on, we assume that $M^{3}$ has constant scalar curvature $R$ and constant mean curvature $H$.

Since from (1.3) $S=6+H^{2}-R$ is also a constant, (1.6) now reads as

$$
\sum_{i, j, k} h_{i j k}^{2}=S(S-3)+H^{2}-H f_{3} .
$$

For our purpose, we also need to express $\sum_{i, j, k, l} h_{i j k l}^{2}$ in terms of $h$ and $\nabla h$. 
To this end, we take the Laplacian of (2.1) to get

$$
\sum_{i, j, k, l} h_{i j k} h_{i j k l l}+\sum_{i, j, k, l} h_{i j k l}^{2}=-\frac{1}{2} H \Delta f_{3} .
$$

On the one hand, we compute

$$
\begin{aligned}
\sum_{i, j, k, l} h_{i j k} h_{i j k l l}= & \sum_{i, j, k, l} h_{i j k} \nabla_{l}\left(h_{i j l k}+2 \sum_{m} h_{m j} R_{m i k l}\right) \\
= & \sum_{i, j, k, l} h_{i j k} h_{i j l k l}+2 \sum_{i, j, k, l, m} h_{i j k} h_{m j l} R_{m i k l} \\
& +2 \sum_{i, j, k, l, m} h_{i j k} h_{m j} \nabla_{l}\left(h_{m k} h_{i l}-h_{m l} h_{i k}\right) \\
= & \sum_{i, j, k, l} h_{i j k}\left(h_{i j l l k}+2 \sum_{m} h_{m j l} R_{m i k l}+\sum_{m} h_{i j m} R_{m l k l}\right) \\
& +2 \sum_{i, j, k, l, m} h_{i j k} h_{m j l} R_{m i k l}+2 \sum_{i, j, k, l, m} h_{i j k} h_{m j}\left(h_{m k l} h_{i l}-h_{m l} h_{i k l}\right) \\
= & \sum_{i, j, k} h_{i j k} \nabla_{k}\left[(3-S) h_{i j}-H \delta_{i j}+H \sum_{m} h_{i m} h_{m j}\right] \\
& +4 \sum_{i, j, k, l, m} h_{i j k} h_{m j l}\left(\delta_{m k} h_{i l}-\delta_{m l} \delta_{i k}+h_{m k} h_{i l}-h_{m l} h_{i k}\right) \\
& +\sum_{i, j, k, l, m} h_{i j k} h_{i j m}\left(2 \delta_{m k}+H h_{m k}-h_{m l} h_{l j}\right) \\
& +2 \sum_{i, j, k} \lambda_{i} \lambda_{j} h_{i j k}^{2}-2 \sum_{i, j, k} \lambda_{i}^{2} h_{i j k}^{2} \\
= & (9-S) \sum_{i, j, k} h_{i j k}^{2}+3 H \sum_{i, j, k} \lambda_{i} h_{i j k}^{2} \\
& +6 \sum_{i, j, k} \lambda_{i} \lambda_{j} h_{i j k}^{2}-3 \sum_{i, j, k} \lambda_{i}^{2} h_{i j k}^{2} .
\end{aligned}
$$

On the other hand, since $\sum_{l} h_{l l i i}=0, \forall i$, and for each $l=1,2,3$,

$$
\begin{aligned}
\frac{1}{3} f_{3, l l} & =\sum_{i, j, k} h_{i j} h_{j k} h_{k i l l}+2 \sum_{i, j, k} h_{i j} h_{j k l} h_{k i l} \\
& =\sum_{i} \lambda_{i}^{2} h_{i i l l}+2 \sum_{i, j} \lambda_{i} h_{i j l}^{2}
\end{aligned}
$$


we. get

$$
\begin{aligned}
\frac{1}{3} \Delta f_{3} & =\sum_{i, l} \lambda_{i}^{2}\left(h_{i i l l}-h_{l l i i}\right)+2 \sum_{i, j, l} \lambda_{i} h_{i j l}^{2} \\
& =\sum_{i, l} \lambda_{i}^{2}\left(\lambda_{i}-\lambda_{l}\right)\left(1+\lambda_{i} \lambda_{l}\right)+2 \sum_{i, j, l} \lambda_{i} h_{i j l}^{2} \\
& =(3-S) f_{3}-H S+H f_{4}+2 \sum_{i, j, k} \lambda_{i} h_{i j k}^{2} .
\end{aligned}
$$

Plugging the above results to (2.2), we find

$$
\begin{aligned}
\sum_{i, j, k, l} h_{i j k l}^{2}=(S-9) & \sum_{i, j, k} h_{i j k}^{2}-6 H \sum_{i, j, k} \lambda_{i} h_{i j k}^{2} \\
-6 \sum_{i, j, k} \lambda_{i} \lambda_{j} h_{i j k}^{2}+ & 3 \sum_{i, j, k} \lambda_{i}^{2} h_{i j k}^{2} \\
& +\frac{3}{2}(S-3) H f_{3}+\frac{3}{2} H^{2}\left(S-f_{4}\right) .
\end{aligned}
$$

To carry out our computations later, it's convenient to introduce $\overline{\lambda_{i}}=\lambda_{i}-\frac{H}{3}$ and accordingly, $\bar{H}=\sum_{i} \overline{\lambda_{i}}, \bar{S}=\sum_{i}{\overline{\lambda_{i}}}^{2}, \overline{f_{k}}=\sum_{i}{\overline{\lambda_{i}}}^{k}, \forall k \geq 3$.

Apparently, $\bar{H}=0$. And it's straightforward to check that $\overline{f_{4}}=\bar{S}^{2} / 2$ and

$$
\begin{aligned}
S & =\bar{S}+\frac{1}{3} H^{2} \\
f_{3} & =\overline{f_{3}}+H \bar{S}+\frac{1}{9} H^{3} \\
f_{4} & =\overline{f_{4}}+\frac{4}{3} H \overline{f_{3}}+\frac{2}{3} H^{2} \bar{S}+\frac{1}{27} H^{4} \\
& \text { etc. }
\end{aligned}
$$

Note that $f_{3}$ and $\overline{f_{3}}$ differ only by a constant.

Let's next interpret (1.3), (2.1) and (2.3) in terms of $\bar{S}$ and $\overline{f_{3}}$.

Lemma 2.1. With the same notations as above, we have

$$
\begin{aligned}
R & =6+\frac{2}{3} H^{2}-\bar{S} \\
\sum_{i, j, k} h_{i j k}^{2} & =\bar{S}(\bar{S}-3)-\frac{1}{3} H^{2} \bar{S}-H \overline{f_{3}} \\
\sum_{i, j, k, l} h_{i j k l}^{2} & =\left(\bar{S}-H^{2}-9\right) \sum_{i, j, k} h_{i j k}^{2}-8 H \sum_{i, j, k} \bar{\lambda}_{i} h_{i j k}^{2}
\end{aligned}
$$




$$
\begin{aligned}
& -6 \sum_{i, j, k} \bar{\lambda}_{i} \bar{\lambda}_{j} h_{i j k}^{2}+3 \sum_{i, j, k} \bar{\lambda}_{i}^{2} h_{i j k}^{2} \\
& +\frac{3}{2} H \bar{f}_{3}\left(\bar{S}-3-\frac{1}{3} H^{2}\right)-\frac{1}{4} H^{2} \bar{S}^{2}
\end{aligned}
$$

Proof. (2.4) and (2.5) follow easily from (1.3) and (2.1), respectively.

As for (2.6), we will employ (2.3). Note that

$$
\begin{aligned}
\sum_{i, j, k} \lambda_{i} h_{i j k}^{2}= & \sum_{i, j, k} \bar{\lambda}_{i} h_{i j k}^{2}+\frac{H}{3} \sum_{i, j, k} h_{i j k}^{2} \\
\sum_{i, j, k} \lambda_{i} \lambda_{j} h_{i j k}^{2}= & \sum_{i, j, k} \bar{\lambda}_{i} \bar{\lambda}_{j} h_{i j k}^{2}+\frac{2 H}{3} \sum_{i, j, k} \bar{\lambda}_{i} h_{i j k}^{2}+\frac{H^{2}}{9} \sum_{i, j, k} h_{i j k}^{2} \\
\sum_{i, j, k} \lambda_{i}^{2} h_{i j k}^{2}= & \sum_{i, j, k} \bar{\lambda}_{i}^{2} h_{i j k}^{2}+\frac{2 H}{3} \sum_{i, j, k} \bar{\lambda}_{i} h_{i j k}^{2}+\frac{H^{2}}{9} \sum_{i, j, k} h_{i j k}^{2} \\
(S-3) f_{3}+H S-H f_{4}= & \left(\bar{S}+\frac{H^{2}}{3}-3\right)\left(\bar{f}_{3}+H \bar{S}+\frac{H^{3}}{9}\right) \\
& +H\left(\bar{S}+\frac{H^{2}}{3}\right)-H\left(\bar{f}_{4}+\frac{4 H \bar{f}_{3}}{3}+\frac{2 H^{2} \bar{S}}{3}+\frac{H^{4}}{27}\right) \\
= & \left(\bar{S}-H^{2}-3\right) \bar{f}_{3}+\left(\frac{1}{2} \bar{S}^{2}-2 \bar{S}-\frac{2}{9} H^{2} \bar{S}\right) H \\
= & \left(\bar{S}-H^{2}-3\right) \bar{f}_{3}+\frac{2}{3}\left(-\frac{1}{4} \bar{S}^{2}+\sum_{i, j, k} h_{i j k}^{2}+H \bar{f}_{3}\right) H \\
= & \left(\bar{S}-\frac{H^{2}}{3}-3\right) \bar{f}_{3}+\frac{2}{3}\left(-\frac{1}{4} \bar{S}^{2}+\sum_{i, j, k} h_{i j k}^{2}\right) H,
\end{aligned}
$$

where we used (2.1) in the second last equality.

It follows from (2.3) that

$$
\begin{aligned}
\sum_{i, j, k, l} h_{i j k l}^{2}= & \left(\bar{S}+\frac{H^{2}}{3}-9\right) \sum_{i, j, k} h_{i j k}^{2}-6 H\left(\sum_{i, j, k} \lambda_{i} h_{i j k}^{2}+\frac{H}{3} \sum_{i, j, k} h_{i j k}^{2}\right) \\
& -6 \sum_{i, j, k} \bar{\lambda}_{i} \bar{\lambda}_{j} h_{i j k}^{2}+3 \sum_{i, j, k} \bar{\lambda}_{i}^{2} h_{i j k}^{2}-2 H \sum_{i, j, k} \lambda_{i} h_{i j k}^{2}-\frac{H^{2}}{3} \sum_{i, j, k} h_{i j k}^{2} \\
& +\frac{3}{2}\left(\bar{S}-\frac{H^{2}}{3}-3\right) H \bar{f}_{3}+\left(-\frac{1}{4} \bar{S}^{2}+\sum_{i, j, k} h_{i j k}^{2}\right) H^{2}
\end{aligned}
$$




$$
\begin{aligned}
= & \left(\bar{S}-H^{2}-9\right) \sum_{i, j, k} h_{i j k}^{2}-8 H \sum_{i, j, k} \lambda_{i} h_{i j k}^{2} \\
& -6 \sum_{i, j, k} \bar{\lambda}_{i} \bar{\lambda}_{j} h_{i j k}^{2}+3 \sum_{i, j, k} \bar{\lambda}_{i}^{2} h_{i j k}^{2}+\frac{3}{2}\left(\bar{S}-\frac{H^{2}}{3}-3\right) H \bar{f}_{3}-\frac{1}{4} H^{2} \bar{S}^{2}
\end{aligned}
$$

This proves Lemma 2.1 .

Now, without loss of generality, we may assume that $H \geq 0$.

Since $M^{3}$ is compact, we can find a point $p \in M^{3}$ such that $\bar{f}_{3}(p)=\max \bar{f}_{3}$ (if $H \leq 0$, we instead consider a minimum point of $\bar{f}_{3}$ ). In particular, $d\left(\sum_{i, j, k} h_{i j} h_{j k} h_{k i}\right)=0$ at $p$.

Note that $d\left(\sum_{i} h_{i i}\right)=0$ and $d\left(\sum_{i, j} h_{i j}^{2}\right)=0$ everywhere, we have that at $p$,

$$
\begin{aligned}
h_{11 l}+h_{22 l}+h_{33 l} & =0 \\
\lambda_{1} h_{11 l}+\lambda_{2} h_{22 l}+\lambda_{3} h_{33 l} & =0 \\
\lambda_{1}^{2} h_{11 l}+\lambda_{2}^{2} h_{22 l}+\lambda_{3}^{2} h_{33 l} & =0
\end{aligned} \quad \forall l=1,2,3
$$

This suggests that the proof would vary according to whether or not the principal curvatures at $p$ are distinct.

Denote by $g(x)$ the number of distinct principal curvatures of $M^{3}$. Clearly, if $g=1$ somewhere, then $\bar{S}=0$ identically and $M^{3}$ is a 3 -sphere. So, in what follows, we assume that $g \geq 2$, i.e. $\bar{S}>0$.

Let $G=\frac{1}{6} \bar{S}^{2}-\frac{1}{\bar{S}} \bar{f}_{3}^{2}$. It's well-known that $G \geq 0$ everywhere and $G=0$ at a point $x \in M^{3}$ if and only if $g=2$ there.

Lemma 2.2. Assume that $\bar{S}>0$. The following equivalent statements hold:

$$
\text { If } R<0 \text {, then } \nabla h \neq 0 \text { everywhere, }
$$

$$
\text { If } \nabla h=0 \text { at a point, then } R \geq 0 \text {. }
$$

Proof. Recall that (2.5)

$$
\sum_{i, j, k} h_{i j k}^{2}=\bar{S}(\bar{S}-3)-\frac{1}{3} H^{2} \bar{S}-H \bar{f}_{3}
$$

Compute that

$$
\mathrm{RHS}=\bar{S}^{2}-3 \bar{S}-\frac{1}{2} H^{2} \bar{S}-\frac{3 \bar{f}_{3}^{2}}{2 \bar{S}}+\frac{1}{6 \bar{S}}\left(H \bar{S}-3 \bar{f}_{3}\right)^{2}
$$




$$
\begin{aligned}
& =\frac{3}{4} \bar{S}^{2}-3 \bar{S}-\frac{1}{2} H^{2} \bar{S}+\frac{3}{2} G+\frac{1}{6 \bar{S}}\left(H \bar{S}-3 \bar{f}_{3}\right)^{2} \\
& =\frac{3}{4}\left(\bar{S}-4-\frac{2}{3} H^{2}\right)+\frac{3}{2} G+\frac{1}{6 \bar{S}}\left(H \bar{S}-3 \bar{f}_{3}\right)^{2} \\
& =\frac{3}{4}(-R) \bar{S}+\frac{3}{2}(\bar{S}+G)+\frac{1}{6 \bar{S}}\left(H \bar{S}-3 \bar{f}_{3}\right)^{2}
\end{aligned}
$$

where in the last equality we used $R=6+\frac{2}{3} H^{2}-\bar{S}$ from (2.4). The assertions then easily follow.

In order to get an idea about how to approach the Main Theorem, let's briefly examine those isoparametric hypersurfaces determined by Cartan.

Suppose that $M^{3}$ is isoparametric. Then the number of distinct principal curvatures $g$ is a constant integer less than or equal to 3 .

From Cartan-Munzner theory [C, M], the distinct principal curvatures of $M^{3}$ are given by

$$
\lambda_{k}=\cot \left(\frac{k-1}{g} \pi+\theta\right), \quad k=1, \ldots, g .
$$

Case (1). If $g=1$, i.e. $\lambda_{k}=\lambda, \forall k=1,2,3$, then,

$$
H=3 \lambda, \quad S=3 \lambda^{2}, \quad R=6+\frac{2}{3} H^{2} \geq 6 .
$$

Case (2). If $g=2$, i.e. $\lambda_{1}=\lambda_{2}=\cot \theta, \lambda_{3}=-\tan \theta$, then

$$
H=2 \cot \theta-\tan \theta, \quad S=2 \cot ^{2} \theta+\tan ^{2} \theta, \quad R=2+2 \cot ^{2} \theta \geq 2 .
$$

It follows from (1.6) that

$$
\sum_{i, j, k} h_{i j k}^{2}=0, \quad \text { i.e. } \quad \nabla h=0 .
$$

Case (3). If $g=3$, i.e. $\lambda_{1}=\lambda, \lambda_{2}=\frac{\sqrt{3}-\lambda}{\sqrt{3} \lambda+1}, \lambda_{3}=\frac{\sqrt{3}+\lambda}{\sqrt{3} \lambda-1}$, then

$$
\begin{aligned}
H & =\frac{\lambda\left(3 \lambda^{2}-7\right)}{3 \lambda^{2}-1} \\
S & =\lambda^{2}+\frac{6 \lambda^{4}+44 \lambda^{2}+6}{\left(3 \lambda^{2}-1\right)^{2}} \\
R & =0
\end{aligned}
$$


Next, in Section 3, we will apply the argument of Peng-Terng in [PT2] to derive the non-negativity of $R$ by contradiction in the case of $g(p)=3$. And in Section 4 , we will extend our study presented in [Ch] to conclude that either $R \geq 6$ or $\nabla h(p)=0$ if $g(p) \leq 2$.

In addition to a great deal of computations on the second fundamental form as in the case of $H=0$, the proof for an arbitrary $H$ also requires techniques on sorting out those extra terms resulted from the presence of the mean curvature.

\section{The Case When $G(p)>0$}

Assume that $\bar{\lambda}_{1}<\bar{\lambda}_{2}<\bar{\lambda}_{3}$. From (2.7), we find

$$
\text { at } p, \quad h_{11 l}=h_{22 l}=h_{33 l}=0, \quad \forall l=1,2,3 .
$$

Suppose that $R<0$. We will derive a contradiction by studying $\nabla h$ and $\nabla^{2} h$ at $p$.

First of all, from (3.1), $\sum_{i, j, k} h_{i j k}^{2}=6 h_{123}^{2}$ at $p$. If $R<0$, it would follow from (2.9) that $h_{123}(p) \neq 0$.

Note that $\nabla \bar{f}_{3}(p)=0$. By differentiating (2.5) and noting (3.1), we would have at $p$,

$$
6 h_{123} h_{123 l}=\sum_{i, j, k} h_{i j k} h_{i j k l}=0, \quad \forall l=1,2,3
$$

and then

$$
h_{123 l}(p)=0, \quad \forall l=1,2,3 .
$$

Moreover, by virtue of the Ricci formulas, it is straightforward to check that $h_{i j k l}$ is symmetric at $p$ as long as $\{1,2,3\} \subset\{i, j, k, l\}$.

From $\sum_{i} h_{i i k l}=0$ and $\sum_{i} \lambda_{i} h_{i i k l}=0, \forall k, l$, everywhere, it would follow that at $p$,

$$
\begin{gathered}
h_{1112}=-h_{2212}-h_{3312}=-h_{2212} \\
\lambda_{1} h_{1112}=-\lambda_{2} h_{2212}-\lambda_{3} h_{3312}=-\lambda_{2} h_{2212}
\end{gathered}
$$

Then, $h_{1112}=h_{2212}=0$ at $p$ since $\lambda_{1} \neq \lambda_{2}$. In general, for each pair $(i, l)$ such that $l \neq i$, we would have $h_{i i i l}(p)=0$. 
Therefore, by virtue of the combination formula (1.7), at $p$,

$$
\sum_{i, j, k, l} h_{i j k l}^{2}=3 \sum_{i, j} h_{i i j j}^{2}-2 \sum_{i} h_{i i i i}^{2} .
$$

Secondly, we have the following:

Observation 3.1. Let $a=\left((\bar{S}-3) / 3-H^{2} / 9\right)$ and $g_{i}=\bar{\lambda}_{i}^{2}-\left(\bar{f}_{3} / \bar{S}\right) \bar{\lambda}_{i}-\bar{S} / 3$.

$$
\text { At } p, \quad h_{i i j j}=\frac{H}{3} \bar{\lambda}_{i}^{2}-a \bar{\lambda}_{i}-\frac{H}{9} \bar{S}+g_{i} \bar{\lambda}_{j}+Z g_{i} g_{j}, \quad \forall i, j,
$$

where $Z$ is a real number to be determined later.

Proof. By definition, $\left(g_{1}, g_{2}, g_{3}\right)$ solves the following under-determined system:

$$
\begin{aligned}
x_{1}+x_{2}+x_{3} & =0 \\
\lambda_{1} x_{1}+\frac{\lambda_{2}}{\lambda_{2}}+\frac{\lambda_{3}}{x_{3}} & =0
\end{aligned}
$$

It is indeed a non-trivial solution since

$$
\begin{aligned}
\sum_{i} g_{i}^{2} & =\sum_{i}\left[\bar{\lambda}_{i}^{4}-\frac{2 \bar{f}_{3}}{\bar{S}} \bar{\lambda}_{i}^{3}+\left(\frac{\bar{f}_{3}^{2}}{\bar{S}^{2}}-\frac{2 \bar{S}}{3}\right) \bar{\lambda}_{i}^{2}-\frac{2 \bar{f}_{3}}{3} \bar{\lambda}_{i}+\frac{\bar{S}^{2}}{9}\right] \\
& =\bar{f}_{4}-\frac{2}{\bar{S}} \bar{f}_{3}^{2}+\left(\frac{\bar{f}_{3}^{2}}{\bar{S}^{2}}-\frac{2 \bar{S}}{3}\right) \bar{S}+\frac{\bar{S}^{2}}{3} \\
& =\frac{1}{2} \bar{S}^{2}-\frac{1}{\bar{S}} \bar{f}_{3}^{2}-\frac{1}{3} \bar{S}^{2} \\
& =G>0
\end{aligned}
$$

Now, set

$$
\tilde{h}_{i i j j}=h_{i i j j}-\frac{H}{3} \bar{\lambda}_{i}^{2}+\frac{H}{9} \bar{S}+a \bar{\lambda}_{i}-g_{i} \bar{\lambda}_{j}, \quad \forall i, j=1,2,3 .
$$

Since at $p, 6 h_{123}^{2}=\sum h_{i j k}^{2}=3 a \bar{S}-H \bar{f}_{3}$, we have $\forall j$,

$$
\sum_{i} \bar{\lambda}_{i} h_{i i j j}=-\sum_{i, k} h_{i k j}^{2}=-2 h_{123}^{2}=-a \bar{S}+\frac{1}{3} H \bar{f}_{3} .
$$

It is then easy to verify that for each $j,\left(\tilde{h}_{11 j j}, \tilde{h}_{22 j j}, \tilde{h}_{33 j j}\right)$ also solves (3.4). Hence, $\forall j, \exists Z_{j} \in R$ s.t. $\forall i, \tilde{h}_{i i j j}=Z_{j} g_{i}$.

Moreover, $\forall i, j, \tilde{h}_{i i j j}=\tilde{h}_{j j i i}$ since

$$
\tilde{h}_{i i j j}-\tilde{h}_{j j i i}=h_{i i j j}-h_{j j i i}-\frac{H}{3}\left(\bar{\lambda}_{i}^{2}-\bar{\lambda}_{j}^{2}\right)+a\left(\bar{\lambda}_{i}-\bar{\lambda}_{j}\right)
$$




$$
\begin{aligned}
& -\left(\bar{\lambda}_{i}^{2}-\frac{\bar{f}_{3}}{\bar{S}} \bar{\lambda}_{i}-\frac{\bar{S}}{3}\right) \bar{\lambda}_{j}+\left(\bar{\lambda}_{j}^{2}-\frac{\bar{f}_{3}}{\bar{S}} \bar{\lambda}_{j}-\frac{\bar{S}}{3}\right) \bar{\lambda}_{i} \\
= & \left(\bar{\lambda}_{i}-\bar{\lambda}_{j}\right)\left[1+\lambda_{i} \lambda_{j}-\frac{H}{3}\left(\bar{\lambda}_{i}+\bar{\lambda}_{j}\right)+a-\left(\bar{\lambda}_{i} \bar{\lambda}_{j}+\frac{\bar{S}}{3}\right)\right] \\
= & 0 .
\end{aligned}
$$

The assertion then follows by setting $Z=(1 / G) \sum_{i} Z_{i} g_{i}$.

Thirdly, having in mind that $n=3$, it is straightforward to verify that

$$
\begin{gathered}
\bar{f}_{4}=\frac{1}{2} \bar{S}^{2}, \quad \bar{f}_{5}=\frac{5}{6} \bar{f}_{3} \bar{S}, \quad \bar{f}_{6}=\frac{1}{3} \bar{f}_{3}^{2}+\frac{1}{4} \bar{S}^{3} \\
\bar{f}_{7}=\frac{7}{12} \bar{f}_{3} \bar{S}^{2}, \quad \bar{f}_{8}=\frac{4}{9} \bar{f}_{3}^{2} \bar{S}+\frac{1}{8} \bar{S}^{4}
\end{gathered}
$$

We then compute that

$$
\begin{aligned}
& \sum_{i} g_{i}^{4}=\sum_{i}\left[\bar{\lambda}_{i}^{8}-4\left(\frac{\bar{f}_{3}}{\bar{S}} \bar{\lambda}_{i}+\frac{\bar{S}}{3}\right) \bar{\lambda}_{i}^{6}+6\left(\frac{\bar{f}_{3}}{\bar{S}} \bar{\lambda}_{i}+\frac{\bar{S}}{3}\right)^{2} \bar{\lambda}_{i}^{4}\right. \\
& \left.-4\left(\frac{\bar{f}_{3}}{\bar{S}} \bar{\lambda}_{i}+\frac{\bar{S}}{3}\right)^{3} \bar{\lambda}_{i}^{2}+\left(\frac{\bar{f}_{3}}{\bar{S}} \bar{\lambda}_{i}+\frac{\bar{S}}{3}\right)^{4}\right] \\
& =\bar{f}_{8}-\frac{4 \bar{f}_{3}}{\bar{S}} \bar{f}_{7}+\left(\frac{6 \bar{f}_{3}^{2}}{\bar{S}^{2}}-\frac{4 \bar{S}}{3}\right) \bar{f}_{6}+\left(4 \bar{f}_{3}-\frac{4 \bar{f}_{3}^{3}}{\bar{S}^{3}}\right) \bar{f}_{5} \\
& +\left(\frac{2 \bar{S}^{2}}{3}-\frac{4 \bar{f}_{3}^{3}}{\bar{S}}+\frac{\bar{f}_{3}^{4}}{\bar{S}^{4}}\right) \bar{f}_{4}+\left(\frac{4 \bar{f}_{3}^{3}}{3 \bar{S}^{2}}-\frac{3 \bar{f}_{3} \bar{S}}{4}\right) \bar{f}_{3}+\frac{2 \bar{f}_{3}^{2} \bar{S}}{3}-\frac{1}{9} \bar{S}^{4} \\
& =\frac{1}{8} \bar{S}^{4}+\frac{4}{9} \bar{f}_{3}^{2} \bar{S}-\frac{4 \bar{f}_{3}}{\bar{S}}\left(\frac{7}{12} \bar{f}_{3} \bar{S}^{2}\right)+\left(\frac{6 \bar{f}_{3}^{2}}{\bar{S}^{2}}-\frac{4 \bar{S}}{3}\right)\left(\frac{\bar{f}_{3}^{2}}{3}+\frac{\bar{S}^{3}}{4}\right) \\
& +\left(4 \bar{f}_{3}-\frac{4 \bar{f}_{3}^{3}}{\bar{S}^{3}}\right)\left(\frac{5}{6} \bar{f}_{3} \bar{S}\right)+\left(\frac{2 \bar{S}^{2}}{3}-\frac{4 \bar{f}_{3}^{3}}{\bar{S}}+\frac{\bar{f}_{3}^{4}}{\bar{S}^{4}}\right)\left(\frac{1}{2} \bar{S}^{2}\right) \\
& +\left(\frac{4 \bar{f}_{3}^{4}}{3 \bar{S}^{2}}-\frac{3}{4} \bar{f}_{3}^{2} \bar{S}\right)+\frac{2}{3} \bar{f}_{3}^{2} \bar{S}-\frac{1}{9} \bar{S}^{4} \\
& =\frac{1}{72} \bar{S}^{4}-\frac{1}{6} \bar{f}_{3}^{2} \bar{S}+\frac{\bar{f}_{3}^{4}}{2 \bar{S}^{2}} \\
& =\frac{1}{2} G^{2} \text {, }
\end{aligned}
$$




$$
\begin{aligned}
& \sum_{i} \bar{\lambda}_{i} g_{i}^{3}=\sum_{i} \bar{\lambda}_{i}\left[\bar{\lambda}_{i}^{6}-3\left(\frac{\bar{f}_{3}}{\bar{S}} \bar{\lambda}_{i}+\frac{\bar{S}}{3}\right) \bar{\lambda}_{i}^{4}+3\left(\frac{\bar{f}_{3}}{\bar{S}} \bar{\lambda}_{i}+\frac{\bar{S}}{3}\right)^{2} \bar{\lambda}_{i}^{2}\right. \\
& \left.-\left(\frac{\bar{f}_{3}}{\bar{S}} \bar{\lambda}_{i}+\frac{\bar{S}}{3}\right)^{3}\right] \\
& =\bar{f}_{7}-\frac{3 \bar{f}_{3}}{\bar{S}} \bar{f}_{6}+\left(\frac{3 \bar{f}_{3}^{2}}{\bar{S}^{2}}-S\right) \bar{f}_{5}+\left(2 \bar{f}_{3}-\frac{\bar{f}_{3}^{2}}{\bar{S}^{2}}\right) \bar{f}_{4} \\
& +\left(\frac{\bar{S}^{2}}{3}-\frac{\bar{f}_{3}^{2}}{\bar{S}}\right) \bar{f}_{3}-\frac{\bar{f}_{3} \bar{S}}{3} \bar{S} \\
& =\frac{7}{12} \bar{f}_{3} \bar{S}^{2}-\frac{3 \bar{f}_{3}}{\bar{S}}\left(\frac{1}{3} \bar{f}_{3}^{2}+\frac{1}{4} \bar{S}^{3}\right)+\left(\frac{3 \bar{f}_{3}^{2}}{\bar{S}^{2}}-S\right)\left(\frac{5}{6} \bar{f}_{3} \bar{S}\right) \\
& +\left(2 \bar{f}_{3}-\frac{\bar{f}_{3}^{2}}{\bar{S}^{2}}\right)\left(\frac{1}{2} \bar{S}^{2}\right)+\left(\frac{\bar{S}^{2} \bar{f}_{3}}{3}-\frac{\bar{f}_{3}^{3}}{\bar{S}}\right)-\frac{1}{3} \bar{f}_{3} \bar{S}^{2} \\
& =0 \text {, } \\
& \sum_{i} \bar{\lambda}_{i}^{2} g_{i}^{2}=\sum_{i} \bar{\lambda}_{i}^{2}\left[\bar{\lambda}_{i}^{4}-\frac{2 \bar{f}_{3}}{\bar{S}} \bar{\lambda}_{i}^{3}+\left(\frac{\bar{f}_{3}^{2}}{\bar{S}^{2}}-\frac{2 \bar{S}}{3}\right) \bar{\lambda}_{i}^{2}+\frac{2}{3} \bar{f}_{3} \bar{\lambda}_{i}+\frac{\bar{S}^{2}}{9}\right] \\
& =\bar{f}_{6}-\frac{2 \bar{f}_{3}}{\bar{S}} \bar{f}_{5}+\left(\frac{\bar{f}_{3}^{2}}{\bar{S}^{2}}-\frac{2 \bar{S}}{3}\right) \bar{f}_{4}+\frac{2}{3} \bar{f}_{3}^{2}+\frac{1}{9} \bar{S}^{3} \\
& =\left(\frac{1}{3} \bar{f}_{3}^{2}+\frac{1}{4} \bar{S}^{3}\right)-\frac{2 \bar{f}_{3}}{\bar{S}}\left(\frac{5}{6} \bar{f}_{3} \bar{S}\right)+\left(\frac{\bar{f}_{3}^{2}}{\bar{S}^{2}}-\frac{2 \bar{S}}{3}\right)\left(\frac{1}{2} \bar{S}^{2}\right)+\frac{2}{3} \bar{f}_{3}^{2}+\frac{1}{9} \bar{S}^{3} \\
& =\frac{1}{6} \bar{S} G \text {, } \\
& \sum_{i} \bar{\lambda}_{i} g_{i}^{2}=\sum_{i} \bar{\lambda}_{i}\left[\bar{\lambda}_{i}^{4}-\frac{2 \bar{f}_{3}}{\bar{S}} \bar{\lambda}_{i}^{3}+\left(\frac{\bar{f}_{3}^{2}}{\bar{S}^{2}}-\frac{2 \bar{S}}{3}\right) \bar{\lambda}_{i}^{2}+\frac{2}{3} \bar{f}_{3} \bar{\lambda}_{i}\right] \\
& =\bar{f}_{5}-\frac{2 \bar{f}_{3}}{\bar{S}} \bar{f}_{4}+\left(\frac{\bar{f}_{3}^{2}}{\bar{S}^{2}}-\frac{2 \bar{S}}{3}\right) \bar{f}_{3}+\frac{2}{3} \bar{f}_{3} \bar{S} \\
& =\frac{5}{6} \bar{f}_{3} \bar{S}-\frac{2 \bar{f}_{3}}{\bar{S}} \frac{1}{2} \bar{S}^{2}+\left(\frac{\bar{f}_{3}^{3}}{\bar{S}^{2}}-\frac{2 \overline{S f}_{3}}{3}\right)+\frac{2}{3} \bar{f}_{3} \bar{S} \\
& =-\frac{\bar{f}_{3}}{\bar{S}} G, \\
& \sum_{i} \bar{\lambda}_{i}^{3} g_{i}=\sum_{i} \bar{\lambda}_{i}^{3}\left(\bar{\lambda}_{i}^{2}-\frac{\bar{f}_{3}}{\bar{S}} \bar{\lambda}_{i}-\frac{\bar{S}}{3}\right)
\end{aligned}
$$




$$
\begin{aligned}
& =\bar{f}_{5}-\frac{\bar{f}_{3}}{\bar{S}} \bar{f}_{4}-\frac{\bar{S}}{3} \bar{f}_{3} \\
& =\frac{5}{6} \bar{f}_{3} \bar{S}-\frac{\bar{f}_{3}}{\bar{S}}\left(\frac{1}{2} \bar{S}^{2}\right)-\frac{1}{3} \bar{f}_{3} \bar{S}=0 \\
\sum_{i} \bar{\lambda}_{i}^{2} g_{i} & =\sum_{i} \bar{\lambda}_{i}^{2}\left(\bar{\lambda}_{i}^{2}-\frac{\bar{f}_{3}}{\bar{S}} \bar{\lambda}_{i}-\frac{\bar{S}}{3}\right) \\
& =\bar{f}_{4}-\frac{\bar{f}_{3}}{\bar{S}} \bar{f}_{3}-\frac{\bar{S}}{3} \bar{S} \\
& =\frac{1}{2} \bar{S}^{2}-\frac{\bar{f}_{3}^{2}}{\bar{S}}-\frac{\bar{S}^{2}}{3}=G .
\end{aligned}
$$

Let $x=Z G$. It follows from (3.3) and the above results that

$$
\begin{aligned}
\sum_{i, j} h_{i i j j}^{2}= & \sum_{i, j}\left(\frac{1}{3} H \bar{\lambda}_{i}^{2}-a \bar{\lambda}_{i}-\frac{1}{9} H \bar{S}+g_{i} \bar{\lambda}_{j}+Z g_{i} g_{j}\right)^{2} \\
= & 3 \sum_{i}\left(\frac{1}{3} H \bar{\lambda}_{i}^{2}-a \bar{\lambda}_{i}-\frac{1}{9} H \bar{S}^{2}\right)^{2}+\sum_{i, j}\left(g_{i} \bar{\lambda}_{j}+Z g_{i} g_{j}\right)^{2} \\
= & 3 \sum_{i}\left[\frac{H^{2} \bar{\lambda}_{i}^{4}}{9}-\frac{2 a H \bar{\lambda}_{i}^{3}}{3}+\left(a^{2}-\frac{2 H^{2} \bar{S}}{27}\right) \bar{\lambda}_{i}^{2}+\frac{2 a H \bar{S}_{i}}{9}+\frac{H^{2} \bar{S}^{2}}{81}\right] \\
& +G \sum_{j}\left(\bar{\lambda}_{j}+Z g_{j}\right)^{2} \\
= & 3\left[\frac{1}{9} H^{2}\left(\frac{1}{2} \bar{S}^{2}\right)-\frac{2 a}{3} H \bar{f}_{3}+\left(a^{2}-\frac{2}{27} H^{2} \bar{S}\right) \bar{S}+\frac{1}{27} H^{2} \bar{S}^{2}\right] \\
& +G\left(\bar{S}^{2}+Z^{2} G\right) \\
= & x^{2}+\bar{S} G+\frac{1}{18} H^{2} \bar{S}^{2}-2 a H \bar{f}_{3}+3 a^{2} \bar{S} \\
= & \sum_{i}\left(\frac{1}{3} H \bar{\lambda}_{i}^{2}-a \bar{\lambda}_{i}-\frac{1}{9} H \bar{S}+g_{i} \bar{\lambda}_{i}+Z g_{i}^{2}\right)^{2} \\
= & \sum_{i}\left(\frac{1}{3} H \bar{\lambda}_{i}^{2}-a \bar{\lambda}_{i}-\frac{1}{9} H \bar{S}\right)^{2}+\sum_{i}\left(g_{i} \bar{\lambda}_{i}+Z g_{i}^{2}\right)^{2} \\
& +2 \sum_{i}\left(\frac{1}{3} H \bar{\lambda}_{i}^{2}-a \bar{\lambda}_{i}-\frac{1}{9} H \bar{S}\right)\left(g_{i} \bar{\lambda}_{i}+Z g_{i}^{2}\right) \\
= & \frac{1}{54} H^{2} \bar{S}^{2}-\frac{2 a}{3} H \bar{f}_{3}+a \bar{S}+\sum_{i}\left(\bar{\lambda}_{i}^{2} g_{i}^{2}+2 Z \bar{\lambda}_{i} g_{i}^{3}+Z^{2} g_{i}^{4}\right) \\
\sum_{i i i i}^{2} &
\end{aligned}
$$




$$
\begin{aligned}
& +2 \sum_{i}\left[\frac{H}{3} \bar{\lambda}_{i}^{3} g_{i}-a \bar{\lambda}_{i}^{2} g_{i}-\frac{H}{9} \overline{S \lambda}_{i} g_{i}\right. \\
& \left.\quad+Z\left(\frac{H}{3} \bar{\lambda}_{i}^{2} g_{i}^{2}-a \bar{\lambda}_{i} g_{i}^{2}-\frac{H}{9} \bar{S} g_{i}^{2}\right)\right] \\
& =\frac{1}{54} H^{2} \bar{S}^{2}-\frac{2 a}{3} H \bar{f}_{3}+a^{2} \bar{S}+\left(\frac{1}{6} \bar{S} G+\frac{1}{2} Z^{2} G^{2}\right) \\
& +2\left\{-a G+Z\left[\frac{1}{3} H\left(\frac{1}{6} \bar{S} G\right)-a\left(-\frac{\bar{f}_{3}}{\bar{S}} G\right)-\frac{1}{9} H \bar{S} G\right]\right\} \\
& =\frac{x^{2}}{2}+\left(\frac{2 a \bar{f}_{3}}{\bar{S}^{2}}-\frac{H \bar{S}}{9}\right) x+\left(\frac{\bar{S}}{6}-2 a\right) G \\
& +\left(\frac{1}{54} H^{2} \bar{S}^{2}-\frac{2}{3} a H \bar{f}_{3}+a^{2} \bar{S}\right)
\end{aligned}
$$

Therefore, from (3.2), at $p$,

$$
\begin{aligned}
\sum_{i, j, k, l} h_{i j k l}^{2}= & 3 \sum_{i, j} h_{i i j j}^{2}-2 \sum_{i} h_{i i i i}^{2} \\
= & 2 x^{2}-4\left(\frac{a \bar{f}_{3}}{\bar{S}}-\frac{H \bar{S}}{18}\right) x \\
& +\left(\frac{8}{3} \bar{S}+4 a\right) G+\frac{7}{54} H^{2} \bar{S}^{2}-\frac{14}{3} a H \bar{f}_{3}+7 a^{2} \bar{S}
\end{aligned}
$$

On the other hand, recall that (2.6)

$$
\begin{aligned}
\sum_{i, j, k, l} h_{i j k l}^{2}=(\bar{S} & \left.-H^{2}-9\right) \sum_{i, j, k} h_{i j k}^{2}-8 H \sum_{i, j, k} \bar{\lambda}_{i} h_{i j k}^{2} \\
& -6 \sum_{i, j, k} \bar{\lambda}_{i} \bar{\lambda}_{j} h_{i j k}^{2}+3 \sum_{i, j, k} \bar{\lambda}_{i}^{2} h_{i j k}^{2} \\
& +\frac{3}{2} H \bar{f}_{3}\left(\bar{S}-3-\frac{1}{3} H^{2}\right)-\frac{1}{4} H^{2} \bar{S}^{2}
\end{aligned}
$$

Since at $p$,

$$
\begin{gathered}
\sum_{i, j, k} h_{i j k}^{2}=3 a \bar{S}-H \bar{f}_{3}, \quad \sum_{i, j, k} \bar{\lambda}_{i} h_{i j k}^{2}=0 \\
\sum_{i, j, k} \bar{\lambda}_{i} \bar{\lambda}_{j} h_{i j k}^{2}=\frac{H}{6} \overline{S f}_{3}-\frac{a}{2} \bar{S}^{2}, \quad \sum_{i, j, k} \bar{\lambda}_{i}^{2} h_{i j k}^{2}=a \bar{S}^{2}-\frac{H}{3} \overline{S f}_{3}
\end{gathered}
$$

the right hand side of (2.6)

$$
R H S=\left(\bar{S}-H^{2}-9\right)\left(3 a \bar{S}-H \bar{f}_{3}\right)-\left(H \overline{S f}_{3}-3 a \bar{S}^{2}\right)+\left(3 a \bar{S}^{2}-H \overline{S f}_{3}\right)
$$




$$
\begin{aligned}
& +\frac{3}{2} H \bar{f}_{3}(3 a)-\frac{1}{4} H^{2} \bar{S}^{2} \\
= & \left(3 \bar{S}-H^{2}-9\right)\left(3 a \bar{S}-H \bar{f}_{3}\right)+\frac{9}{2} a H \bar{f}_{3}-\frac{1}{4} H^{2} \bar{S}^{2} \\
= & (9 a)\left(3 a \bar{S}-H \bar{f}_{3}\right)+\frac{9}{2} a H \bar{f}_{3}-\frac{1}{4} H^{2} \bar{S}^{2} \\
= & 27 a^{2} \bar{S}-\frac{9}{2} a H \bar{f}_{3}-\frac{1}{4} H^{2} \bar{S}^{2}
\end{aligned}
$$

Therefore, (2.6) would read as

$$
x^{2}-2\left(\frac{a \bar{f}_{3}}{\bar{S}}-\frac{H \bar{S}}{18}\right) x=C
$$

where

$$
C=10 a^{2} \bar{S}+\frac{1}{12} a H \bar{f}_{3}-\frac{41}{216} H^{2} \bar{S}^{2}-\left(\frac{4}{3} \bar{S}+2 a\right) G
$$

And the maximality of $\bar{f}_{3}$ at $p$ would imply that for each $l=1,2,3$,

$$
\begin{aligned}
0 \geq \frac{1}{3} \bar{f}_{3, l l}= & \sum_{i} \lambda_{i}^{2} h_{i i l l}+2 \sum_{i, j} \lambda_{i} h_{i j l}^{2} \\
= & \sum_{i}\left(\bar{\lambda}_{i}^{2}+\frac{2 H}{3} \lambda_{i}\right) h_{i i l l}+2\left(H-\lambda_{l}\right) h_{123}^{2} \\
= & \sum_{i} \bar{\lambda}_{i}^{2}\left(\frac{H}{3} \bar{\lambda}_{i}^{2}-a \bar{\lambda}_{i}-\frac{H \bar{S}}{9}+g_{i} \bar{\lambda}_{l}+Z g_{i} g_{l}\right) \\
& \quad-\frac{2 H}{3} \sum_{i, j} h_{i j l}^{2}+2\left(\frac{2}{3} H-\bar{\lambda}_{l}\right) h_{123}^{2} \\
= & x g_{l}+\left(G-a \bar{S}+\frac{1}{3} H \bar{f}_{3}\right) \bar{\lambda}_{l}+\frac{1}{18} H \bar{S}^{2}-a \bar{f}_{3}
\end{aligned}
$$

As in the Peng-Terng's proof [PT2] of their theorem, we will derive a contradiction by exploiting equation (3.5) and inequalities (3.6). The crucial step is to obtain a lower bound of $C$ so that we can have a neat estimate on $x$.

Summing up the inequalities (3.6) over $l=1,2,3$, we have at $p$,

$$
0 \geq \frac{1}{3} \Delta \bar{f}_{3}=\frac{1}{6} H \bar{S}^{2}-3 a \bar{f}_{3}
$$


Denote $\alpha=-\frac{1}{3 \bar{S}} \Delta \bar{f}_{3}(p)$ and note that if $R<0$,

$$
a=\frac{S-3}{3}-\frac{1}{9} H^{2}=\frac{1}{6}(\bar{S}-R)>0,
$$

we would rewrite (3.7) as

$$
0 \leq \alpha=\frac{3 a}{\bar{S}} \bar{f}_{3}-\frac{H}{6} \bar{S} \quad\left(=\frac{\bar{f}_{3}}{2}-\frac{H \bar{S}}{6}-\frac{R \bar{f}_{3}}{2 \bar{S}}\right) .
$$

Since

$$
\begin{aligned}
G-a \bar{S}+\frac{H}{3} \bar{f}_{3} & =\left(\frac{\bar{S}^{2}}{6}-\frac{\bar{f}_{3}^{2}}{\bar{S}}\right)-\frac{1}{6}(\bar{S}-R) \bar{S}+\frac{H}{3} \bar{f}_{3} \\
& =\frac{R}{6} \bar{S}-\frac{2 \bar{f}_{3}}{\bar{S}}\left[\left(\frac{\bar{f}_{3}}{2}-\frac{H \bar{S}}{6}-\frac{R \bar{f}_{3}}{2 \bar{S}}\right)+\frac{R \bar{f}_{3}}{2 \bar{S}}\right] \\
& =\frac{R}{\bar{S}} G-\frac{2 \alpha}{\bar{S}} \bar{f}_{3},
\end{aligned}
$$

we would claim that

$$
\begin{aligned}
C & =10 a\left(a \bar{S}-\frac{1}{3} H \bar{f}_{3}-G\right)+\frac{41}{36} H \bar{S}\left(\frac{3 a}{\bar{S}} \bar{f}_{3}-\frac{H}{6} \bar{S}\right)+\left(8 a-\frac{4}{3} \bar{S}\right) G \\
& =10 a\left(\frac{2 \alpha}{\bar{S}} \bar{f}_{3}-\frac{R}{\bar{S}} G\right)+\frac{41}{36} H \bar{S} \alpha+\frac{4}{3}(-R) G \\
& =\alpha\left[\left(\frac{20 a}{\bar{S}} \bar{f}_{3}-\frac{10}{9} H \bar{S}\right)+\frac{9}{4} H \bar{S}\right]+\left(\frac{10 a}{\bar{S}}+\frac{4}{3}\right)(-R) G \\
& =\frac{20}{3} \alpha^{2}+\beta
\end{aligned}
$$

where $\beta=\frac{9 \alpha}{4} H \bar{S}+\left(\frac{10 a}{\bar{S}}+\frac{4}{3}\right)(-R) G>0$ if $R<0$.

Therefore, from (3.5) we would have $x^{2}-\frac{2 \alpha}{3} x>\frac{20}{3} \alpha^{2}$, that is

$$
\text { either } x>\frac{1+\sqrt{61}}{3} \alpha \quad\left(\geq \frac{8}{3} \alpha\right), \quad \text { or } \quad x<\frac{1-\sqrt{61}}{3} \alpha \quad(\leq-2 \alpha) \text {. }
$$

However, note that at $p, \bar{f}_{3}=\frac{H \bar{S}^{2}}{18 a}-\frac{\Delta \bar{f}_{3}}{9 a} \geq 0$, we may write $\bar{f}_{3}=\bar{S} \sqrt{\frac{\bar{S}}{6}} \cos \theta$ with $\theta \in\left(0, \frac{\pi}{2}\right]$, and from the theory of cubic equation, $\bar{\lambda}_{l}=2 \sqrt{\frac{\bar{S}}{6}} \cos \frac{\theta+2 \pi l}{3}$, 
$l=1,2,3$. In particular,

$$
\overline{\lambda_{1}}<\overline{\lambda_{2}}<0, \quad \frac{\bar{S}}{6}<{\overline{\lambda_{1}}}^{2} \leq \frac{\bar{S}}{2}, \quad 0 \leq{\overline{\lambda_{2}}}^{2}<\frac{\bar{S}}{6}
$$

Note also that for each fixed $l=1,2,3, \bar{f}_{3}=3 \bar{\lambda}_{l}\left(\bar{\lambda}_{l}^{2}-\bar{S} / 2\right)$ and then

$$
\begin{aligned}
g_{l} & =\bar{\lambda}_{l}^{2}-\frac{\bar{f}_{3}}{\bar{S}} \bar{\lambda}_{l}-\frac{\bar{S}}{3} \\
& =\bar{\lambda}_{l}^{2}-\frac{3}{\bar{S}} \bar{\lambda}_{l}^{2}\left(\bar{\lambda}_{l}^{2}-\frac{\bar{S}}{2}\right)-\frac{\bar{S}}{3} \\
& =\frac{3}{\bar{S}}\left(\bar{\lambda}_{l}^{2}-\frac{\bar{S}}{6}\right)\left(\frac{2 \bar{S}}{3}-\bar{\lambda}_{l}^{2}\right)
\end{aligned}
$$

Now, if $x>8 \alpha / 3$, by taking $l=1$ in (3.6), we would find

$$
\begin{array}{rl}
0 & x\left(\frac{3}{\bar{S}}\right)\left(\bar{\lambda}_{1}^{2}-\frac{\bar{S}}{6}\right)\left(\frac{2 \bar{S}}{3}-\bar{\lambda}_{1}^{2}\right)+\left(\frac{R}{\bar{S}} G-\frac{2 \alpha}{\bar{S}} \bar{f}_{3}\right) \bar{\lambda}_{1}-\frac{\alpha}{3} \bar{S} \\
> & \frac{8}{3} \alpha\left(\frac{3}{\bar{S}}\right)\left(\bar{\lambda}_{1}^{2}-\frac{\bar{S}}{6}\right)\left(\frac{2 \bar{S}}{3}-\bar{\lambda}_{1}^{2}\right)+\frac{G}{\bar{S}}(-R)\left(-\bar{\lambda}_{1}\right) \\
& \quad-\frac{2 \alpha}{\bar{S}}\left[3 \bar{\lambda}_{1}^{2}\left(\bar{\lambda}_{1}^{2}-\frac{\bar{S}}{2}\right)+\frac{\bar{S}^{2}}{6}\right] \\
> & \frac{\alpha}{\bar{S}}\left(\bar{\lambda}_{1}^{2}-\frac{\bar{S}}{6}\right)\left(\frac{11 \bar{S}}{3}-7 \bar{\lambda}_{1}^{2}\right)
\end{array}
$$

It would follow that ${\overline{\lambda_{1}}}^{2}>11 \bar{S} / 21$, contradicting to ${\overline{\lambda_{1}}}^{2} \leq \bar{S} / 2$ in (3.8).

Similarly, if $x<-2 \alpha$, taking $l=2$ in (3.6) would lead to $0>2 \alpha\left(\frac{\bar{S}}{6}-{\overline{\lambda_{2}}}^{2}\right)$, another contradiction to ${\overline{\lambda_{2}}}^{2}<\bar{S} / 6$ in (3.8). This establishes that $R \geq 0$ in the case when $G(p)>0$.

\section{The CASE When $G(p)=0$}

As indicated by the computation on isoparametric hypersurfaces in Section 2 , we expect to have $\nabla h$ vanishing at $p$. It in turn follows from (2.10) that $R \geq 0$ if $\nabla h$ vanishes at $p$.

Suppose now that at $p \in M^{3}, \lambda_{1}=\lambda_{2}$. We will investigate the second fundamental form $h$ and its covariant derivatives at $p$ to show that either $R>6$ or $\sum_{i, j, k} h_{i j k}^{2}(p)=0$. 
Without loss of generality, we may assume that at $p$,

$$
\left(h_{i j}\right)=\frac{H}{3} I_{3}+\left(\begin{array}{lll}
\bar{\lambda} & & \\
& \bar{\lambda} & \\
& & -2 \bar{\lambda}
\end{array}\right) \text { with } 6 \bar{\lambda}^{2}=\bar{S}>0,
$$

where $I_{3}$ denotes the identity matrix of rank 3 .

Let's next study the covariant derivative $\nabla h$ of $h$ at $p$. Recall that from (2.7), for each $l=1,2,3$,

$$
h_{11 l}+h_{22 l}+h_{33 l}=0, \quad \lambda_{1} h_{11 l}+\lambda_{2} h_{22 l}+\lambda_{3} h_{33 l}=0 .
$$

It follows that at $p$,

$$
h_{22 l}=-h_{11 l}, \quad h_{33 l}=0, \quad \forall l=1,2,3 .
$$

And since at $p,\left\{e_{1}, e_{2}\right\}$ is a basis of the $\lambda$-eigenspace of $\left(h_{i j}\right)$, we may rotate it if necessary to have

$$
h_{123}(p)=0 .
$$

Recall also that from (2.5),

$$
\sum_{i, j, k} h_{i j k}^{2}=\bar{S}(\bar{S}-3)-\frac{1}{3} H^{2} \bar{S}-H \bar{f}_{3} .
$$

Since now $\bar{f}_{3}(p)=-\overline{\lambda S}$, we have

$$
\text { at } p, \quad \sum_{i, j, k} h_{i j k}^{2}=\bar{S}(\bar{S}-3)-\frac{1}{3} H^{2} \bar{S}+\bar{\lambda} H \bar{S} .
$$

Note that by virtue of (4.2) and (4.3), we may express $\nabla h$ at $p$ in terms of $h_{111}, h_{112}$ and $h_{113}$. We can compute out the left hand side of (4.4) in this way by using (1.7) as follows:

$$
\begin{aligned}
\sum_{i, j, k} h_{i j k}^{2} & =6 h_{123}^{2}+\sum_{i} h_{i i i}^{2}+3 \sum_{i \neq k} h_{i i k}^{2} \\
& =\left(h_{111}^{2}+h_{222}^{2}\right)+3\left(h_{112}^{2}+h_{113}^{2}+h_{221}^{2}+h_{223}^{2}\right) \\
& =6 h_{113}^{2}+4\left(h_{111}^{2}+h_{112}^{2}\right)
\end{aligned}
$$

Denote $a=h_{113}^{2}(p) \geq 0, b=h_{111}^{2}(p)+h_{112}^{2}(p) \geq 0$, then (4.4) reads as

$$
6 a+4 b=\bar{S}(\bar{S}-3)-\frac{1}{3} H^{2} \bar{S}+\bar{\lambda} H \bar{S} .
$$


Hence, in order to have $\nabla h$ vanishes at $p$, it suffices to show that $a=b=0$. We will show that either $R \geq 6$ or $a=b=0$ by studying the higher covariant derivatives of $h$.

Lemma 4.1. At $p$,

(1) $h_{i j k l}$ is symmetric in all the indices except for those permutations $\{i, j, k, l\}$ of either $\{1,1,3,3\}$ or $\{2,2,3,3\}$.

(2) $h_{3311}=h_{3322}=\frac{2}{3 \bar{\lambda}}(a+b), \quad h_{3333}=\frac{2 a}{3 \bar{\lambda}}, \quad h_{3312}=0$, $h_{3313}=\frac{2}{3 \bar{\lambda}} h_{111} h_{113}, \quad h_{3323}=\frac{2}{3 \bar{\lambda}} h_{112} h_{113}$, and $h_{1111}=h_{2222}, \quad h_{1133}=h_{2233}=-\frac{a}{3 \bar{\lambda}}$.

Proof. (1) simply follows from the Ricci formula: $\forall i, j, k, l$,

$$
\begin{aligned}
h_{i j k l}-h_{i j l k} & =\sum_{m}\left(h_{m j} R_{m i k l}+h_{i m} R_{m j k l}\right) \\
& =\left(\lambda_{i}-\lambda_{j}\right)\left(1+\lambda_{i} \lambda_{j}\right)\left(\delta_{i k} \delta_{j l}-\delta_{i l} \delta_{j k}\right)
\end{aligned}
$$

To see (2), we differentiate $\sum_{i} h_{i i}=$ const. and $\sum_{i, j} h_{i j}^{2}=$ const. twice to get

$$
\begin{aligned}
h_{11 k l}+h_{22 k l}+h_{33 k l} & =0, \\
\sum_{i, j}\left(h_{i j k} h_{i j l}+h_{i j} h_{i j k l}\right) & =0, \quad \forall k, l
\end{aligned}
$$

Evaluating the above equations at $p$ and using (4.1), we have

$$
3 \bar{\lambda} h_{33 k l}=\sum_{i, j} h_{i j k} h_{i j l}, \quad \forall k, l .
$$

This yields the first six equalities by explicitly writing out all terms on the right hand side for all pairs $(k, l)$ and applying (4.2) and (4.3).

In turn, we find $h_{1111}=-h_{2211}-h_{3311}=-h_{1122}-h_{3322}=h_{2222}$.

Finally, employing the Ricci formula again, we compute that

$$
\begin{aligned}
h_{1133}=h_{1313} & =h_{1331}+\left(\lambda_{1}-\lambda_{3}\right)\left(1+\lambda_{1} \lambda_{3}\right) \\
& =\frac{2}{3 \bar{\lambda}}(a+b)+3 \bar{\lambda}\left[1+\left(\frac{1}{3} H+\bar{\lambda}\right)\left(\frac{1}{3} H-2 \bar{\lambda}\right)\right] \\
& =\frac{2}{3 \bar{\lambda}}(a+b)+\frac{\bar{S}}{2 \bar{\lambda}}\left(1+\frac{1}{9} H^{2}-\frac{1}{3} H \bar{\lambda}-\frac{1}{3} \bar{S}\right) \\
& =\frac{2}{3 \bar{\lambda}}(a+b)+\frac{1}{6 \bar{\lambda}}\left(\bar{S}(3-\bar{S})+\frac{1}{3} H^{2} \bar{S}-\bar{\lambda} H \bar{S}\right)
\end{aligned}
$$




$$
\begin{aligned}
& =\frac{2}{3 \bar{\lambda}}(a+b)-\frac{1}{6 \bar{\lambda}}(6 a+4 b) \\
& =-\frac{a}{3 \bar{\lambda}}
\end{aligned}
$$

And $h_{2233}=-a /(3 \bar{\lambda})$ follows in the same way, or from $h_{2233}=-\left(h_{1133}+h_{3333}\right)$. This proves Lemma 4.1 .

Immediately, we have the following:

Corollary 4.2. At $p$,

$$
\begin{gathered}
\sum_{\alpha, \beta} h_{\alpha \beta 33}^{2}=\frac{4 a^{2}}{3 \bar{S}}, \quad \sum_{\alpha, \beta} h_{33 \alpha \beta}^{2}=\frac{16}{3 \bar{S}}(a+b)^{2} \\
\sum_{\alpha} h_{\alpha 333}^{2}=\frac{8 a b}{3 \bar{S}}, \quad h_{3333}^{2}=\frac{8 a^{2}}{3 \bar{S}}
\end{gathered}
$$

here and afterforth, we use $\alpha, \beta, \gamma$, etc., to denote indices ranging from 1 to 2.

For later use, we perform here the following computations at $p$.

$$
\begin{aligned}
\sum_{i, j, k} \bar{\lambda}_{i} h_{i j k}^{2} & =\bar{\lambda} \sum_{\alpha, j, k} h_{\alpha j k}^{2}+(-2 \bar{\lambda}) \sum_{j, k} h_{3 j k}^{2} \\
& =\bar{\lambda}(4 b+4 a)-2 \bar{\lambda}(2 a) \\
& =4 b \bar{\lambda} \\
\sum_{i, j, k} \bar{\lambda}_{i} \bar{\lambda}_{j} h_{i j k}^{2} & =\bar{\lambda}^{2} \sum_{\alpha, \beta, k} h_{\alpha \beta k}^{2}+2 \bar{\lambda}(-2 \bar{\lambda}) \sum_{\alpha, k} h_{3 \alpha k}^{2} \\
& =\bar{\lambda}^{2}(4 b+2 a)-4 \bar{\lambda}^{2}(2 a) \\
& =-a \bar{S}+\frac{2}{3} b \bar{S}, \\
\sum_{i, j, k} \bar{\lambda}_{i}^{2} h_{i j k}^{2} & =\bar{\lambda}^{2} \sum_{\alpha, j, k} h_{\alpha j k}^{2}+(-2 \bar{\lambda})^{2} \sum_{j, k} h_{3 j k}^{2} \\
& =\bar{\lambda}^{2}(4 b+4 a)+4 \bar{\lambda}^{2}(2 a) \\
& =2 a \bar{S}+\frac{2}{3} b \bar{S} .
\end{aligned}
$$

In summary, we have 
Lemma 4.3. At $p$,

$$
\begin{aligned}
\sum_{i, j, k} \bar{\lambda}_{i} h_{i j k}^{2} & =4 b \bar{\lambda} \\
\sum_{i, j, k} \bar{\lambda}_{i} \bar{\lambda}_{j} h_{i j k}^{2} & =-a \bar{S}+\frac{2}{3} b \bar{S} \\
\sum_{i, j, k} \bar{\lambda}_{i}^{2} h_{i j k}^{2} & =2 a \bar{S}+\frac{2}{3} b \bar{S}
\end{aligned}
$$

We proceed to study $\left\|\nabla^{2} h\right\|^{2}$ at $p$. Recall that from (2.6)

$$
\begin{aligned}
\sum_{i, j, k, l} h_{i j k l}^{2}=(\bar{S}- & \left.H^{2}-9\right) \sum_{i, j, k} h_{i j k}^{2}-8 H \sum_{i, j, k} \bar{\lambda}_{i} h_{i j k}^{2} \\
& -6 \sum_{i, j, k} \bar{\lambda}_{i} \bar{\lambda}_{j} h_{i j k}^{2}+3 \sum_{i, j, k} \bar{\lambda}_{i}^{2} h_{i j k}^{2} \\
& +\frac{3}{2} H \bar{f}_{3}\left(\bar{S}-3-\frac{1}{3} H^{2}\right)-\frac{1}{4} H^{2} \bar{S}^{2}
\end{aligned}
$$

Now at $p$, by virtue of Lemma 4.1 , Corollary 4.2 and Lemma 4.3 ,

$$
\begin{aligned}
L H S= & \sum_{\alpha, \beta, \gamma, \sigma} h_{\alpha \beta \gamma \sigma}^{2}+4 \sum_{\alpha, \beta, \gamma} h_{\alpha \beta \gamma 3}^{2}+3 \sum_{\alpha, \beta}\left(h_{\alpha \beta 33}^{2}+h_{33 \alpha \beta}^{2}\right) \\
& \quad+4 \sum_{\alpha} h_{\alpha 333}^{2}+h_{3333}^{2} \\
= & \sum_{\alpha, \beta, \gamma, \sigma} h_{\alpha \beta \gamma \sigma}^{2}+4 \sum_{\alpha, \beta, \gamma} h_{\alpha \beta \gamma 3}^{2}+3\left(\frac{4}{3 \bar{S}} a^{2}+\frac{16}{3 \bar{S}}(a+b)^{2}\right) \\
& \quad+\frac{32}{3 \bar{S}} a b+\frac{8}{3 \bar{S}} a^{2} \\
= & \sum_{\alpha, \beta, \gamma, \sigma} h_{\alpha \beta \gamma \sigma}^{2}+4 \sum_{\alpha, \beta, \gamma} h_{\alpha \beta \gamma 3}^{2}+\frac{4}{3 \bar{S}}\left(17 a^{2}+32 a b+12 b^{2}\right) \\
R H S= & \left(\bar{S}-H^{2}-9\right)(6 a+4 b)-8 H(4 b \bar{\lambda})-6\left(-a \bar{S}+\frac{2}{3} b \bar{S}\right) \\
& \quad+3\left(2 a \bar{S}+\frac{2}{3} b \bar{S}\right)+\frac{3}{2} H(-\overline{\lambda S})\left(\frac{6 a+4 b}{\bar{S}}-\bar{\lambda} H\right)-\frac{1}{4} H^{2} \bar{S}^{2} \\
= & 3 a\left(6 \bar{S}-18-2 H^{2}-3 H \bar{\lambda}\right)+2 b\left(\bar{S}-18-2 H^{2}-19 H \bar{\lambda}\right) .
\end{aligned}
$$

Therefore, we have the following: 
Lemma 4.4. At $p$,

$$
\begin{aligned}
\sum_{\alpha, \beta, \gamma} h_{\alpha \beta \gamma 3}^{2}= & -\frac{1}{4} \sum_{\alpha, \beta, \gamma, \sigma} h_{\alpha \beta \gamma \sigma}^{2}-\frac{1}{3 \bar{S}}\left(17 a^{2}+32 a b+12 b^{2}\right) \\
& +\frac{3 a}{4}\left(6 \bar{S}-18-2 H^{2}-3 H \bar{\lambda}\right)+\frac{b}{2}\left(\bar{S}-18-2 H^{2}-19 H \bar{\lambda}\right)
\end{aligned}
$$

Due to the lack of constraints among the quantities $a, b, \sum h_{\alpha \beta \gamma \sigma}^{2}$ and $\sum h_{\alpha \beta \gamma 3}^{2}$, we next have to appeal to $\nabla^{3} h$. First of all, by differentiating $\sum_{i, j} h_{i j}^{2}=S$ triply, we get

$$
\sum_{i, j}\left(h_{i j} h_{i j k l m}+h_{i j k} h_{i j l m}+h_{i j l} h_{i j k m}+h_{i j m} h_{i j k l}\right)=0, \quad \forall k, l, m .
$$

Again, since at $p$,

$$
\begin{aligned}
\sum_{i, j} h_{i j} h_{i j k l m} & =\left(\frac{H}{3}+\bar{\lambda}\right)\left(h_{11 k l m}+h_{22 k l m}\right)+\left(\frac{H}{3}-2 \bar{\lambda}\right) h_{33 k l m} \\
& =-3 \bar{\lambda} h_{33 k l m}
\end{aligned}
$$

we have at $p$

$$
3 \bar{\lambda} h_{33 k l m}=\sum_{i, j}\left(h_{i j k} h_{i j l m}+h_{i j l} h_{i j k m}+h_{i j m} h_{i j k l}\right), \quad \forall k, l, m .
$$

It follows that at $p$,

$$
\bar{\lambda} \sum_{k, l, m} h_{k l m} h_{33 k l m}=\sum_{i, j, k, l, m} h_{k l m} h_{i j k} h_{i j l m} .
$$

Compute the right hand side of the above equation

$$
\begin{aligned}
\mathrm{RHS}= & \sum_{k, l, m} h_{k l m}\left(h_{11 k} h_{11 l m}+h_{22 k} h_{22 l m}+2 h_{12 k} h_{12 l m}\right. \\
& \left.\quad+2 h_{13 k} h_{13 l m}+2 h_{23 k} h_{23 l m}\right) \\
= & \sum_{k, l, m} h_{11 k} h_{k l m}\left(h_{11 l m}-h_{22 l m}\right)+2 \sum_{l, m}\left(h_{112} h_{1 l m}-h_{111} h_{2 l m}\right) h_{12 l m} \\
\quad & \quad+2 \sum_{l, m} h_{1 l m} h_{113} h_{13 l m}+2 \sum_{l, m} h_{2 l m} h_{223} h_{23 l m} \\
= & \sum_{k} h_{11 k}\left[h_{k 11}\left(h_{1111}-h_{2211}\right)+h_{k 22}\left(h_{1122}-h_{2222}\right)\right. \\
& \left.+2 h_{k 12}\left(h_{1112}-h_{2212}\right)+2 h_{k 13}\left(h_{1113}-h_{2213}\right)+2 h_{k 23}\left(h_{1123}-h_{2223}\right)\right] \\
& +2 h_{112}\left(h_{111} h_{1211}+h_{122} h_{1222}+2 h_{112} h_{1212}+2 h_{113} h_{1213}\right)
\end{aligned}
$$




$$
\begin{aligned}
& -2 h_{111}\left(h_{211} h_{1211}+h_{222} h_{1222}+2 h_{122} h_{1212}+2 h_{223} h_{1223}\right) \\
& +2 h_{113}\left[h_{111} h_{1113}+h_{122} h_{1322}+2 h_{112} h_{1312}+h_{113}\left(h_{1133}+h_{3311}\right)\right] \\
& -2 h_{113}\left[h_{211} h_{1123}+h_{222} h_{2322}+2 h_{212} h_{2312}+h_{223}\left(h_{2233}+h_{3322}\right)\right] \\
= & 2(a+b)\left(h_{1111}-h_{2211}\right)+4 b h_{1122} \\
& -4 h_{113}\left(h_{111} h_{3313}+h_{112} h_{3323}\right)+2 a\left(h_{1133}+h_{3311}+h_{2233}+h_{3322}\right) \\
= & 4 a h_{1111}+2(a-b) \frac{2(a+b)}{3 \bar{\lambda}}-4 a \frac{2 b}{3 \bar{\lambda}}+4 a \frac{a+2 b}{3 \bar{\lambda}} \\
= & 4 a h_{1111}+\frac{8 a^{2}-4 b^{2}}{3 \bar{\lambda}},
\end{aligned}
$$

we obtain that

$$
\sum_{k, l, m} h_{k l m} h_{33 k l m}=\frac{4 a}{\bar{\lambda}} h_{1111}+\frac{16 a^{2}-8 b^{2}}{\bar{S}} .
$$

Secondly, by differentiating $\sum_{i, j, k} h_{i j k}^{2}=\bar{S}(\bar{S}-3)-\frac{1}{3} H^{2} \bar{S}-H \bar{f}_{3}$ twice w.r.t. $e_{3}$,

$$
\sum_{i, j, k}\left(h_{i j k} h_{i j k 33}+h_{i j k 3}^{2}\right)=-\frac{1}{2} H \nabla_{e_{3} e_{3}}^{2} \bar{f}_{3} .
$$

Since at $p$,

$$
\begin{aligned}
\nabla_{e_{3} e_{3}}^{2} \bar{f}_{3} & =3 \sum_{i} \lambda_{i}^{2} h_{i i 33}+6 \sum_{i, j} \lambda_{i} h_{i j 3}^{2} \\
& =3\left[\left(\bar{\lambda}+\frac{H}{3}\right)^{2}\left(-\frac{1}{3 \bar{\lambda}} a-\frac{1}{3 \bar{\lambda}} a\right)+\left(-2 \bar{\lambda}+\frac{H}{3}\right)^{2}\left(\frac{2}{3 \bar{\lambda}} a\right)\right]+6\left(\bar{\lambda}+\frac{H}{3}\right)(2 a) \\
& =18 \bar{\lambda} a
\end{aligned}
$$

and from Corollary 4.2 and Lemma 4.4,

$$
\begin{aligned}
\sum_{i, j, k} h_{i j k 3}^{2}= & \sum_{\alpha, \beta, \gamma} h_{\alpha \beta \gamma 3}^{2}+3 \sum_{\alpha, \beta} h_{\alpha \beta 33}^{2}+3 \sum_{\alpha} h_{\alpha 333}^{2}+h_{3333}^{2} \\
= & -\frac{1}{4} \sum_{\alpha, \beta, \gamma, \sigma} h_{\alpha \beta \gamma \sigma}^{2}-\frac{1}{3 \bar{S}}\left(17 a^{2}+32 a b+12 b^{2}\right) \\
& +\frac{3 a}{4}\left(6 \bar{S}-18-2 H^{2}-3 H \bar{\lambda}\right)+\frac{b}{2}\left(\bar{S}-18-2 H^{2}-19 H \bar{\lambda}\right) \\
& +\frac{4}{\bar{S}} a^{2}+\frac{8}{\bar{S}} a b+\frac{8}{3 \bar{S}} a^{2} \\
= & -\frac{1}{4} \sum_{\alpha, \beta, \gamma, \sigma} h_{\alpha \beta \gamma \sigma}^{2}+\frac{1}{3 \bar{S}}\left(3 a^{2}-8 a b-12 b^{2}\right)
\end{aligned}
$$




$$
+\frac{3 a}{4}\left(6 \bar{S}-18-2 H^{2}-3 H \bar{\lambda}\right)+\frac{b}{2}\left(\bar{S}-18-2 H^{2}-19 H \bar{\lambda}\right)
$$

we have that at $p$,

$$
\begin{aligned}
\sum_{i, j, k} h_{i j k} h_{i j k 33}=\frac{1}{4} & \sum_{\alpha, \beta, \gamma, \sigma} h_{\alpha \beta \gamma \sigma}^{2}-\frac{1}{3 \bar{S}}\left(3 a^{2}-8 a b-12 b^{2}\right) \\
& -\frac{3 a}{4}\left(6 \bar{S}-18-2 H^{2}+9 H \bar{\lambda}\right) \\
& -\frac{b}{2}\left(\bar{S}-18-2 H^{2}-19 H \bar{\lambda}\right)
\end{aligned}
$$

By substracting (4.7) from (4.6) we find

$$
\begin{aligned}
\sum_{i, j, k} h_{i j k}\left(h_{33 i j k}-h_{i j k 33}\right)=\frac{4 a}{\bar{\lambda}} & h_{1111}-\frac{1}{4} \sum_{\alpha, \beta, \gamma, \sigma} h_{\alpha \beta \gamma \sigma}^{2} \\
& +\frac{1}{3 \bar{S}}\left(51 a^{2}-8 a b-36 b^{2}\right) \\
& +\frac{3 a}{4}\left(6 \bar{S}-18-2 H^{2}+9 H \bar{\lambda}\right) \\
& +\frac{b}{2}\left(\bar{S}-18-2 H^{2}-19 H \bar{\lambda}\right)
\end{aligned}
$$

Finally, by using the Ricci formulas and Lemmas 4.1 and 4.3, we compute at $p$

$$
\begin{aligned}
& \sum_{i, j, k} h_{i j k}\left(h_{33 i j k}-h_{i j k 33}\right) \\
& =\sum_{i, j, k} h_{i j k} \nabla_{e_{k}}\left(h_{3 i j 3}+\sum_{m} h_{m i} R_{m 33 j}+\sum_{m} h_{3 m} R_{m i 3 j}\right) \\
& \quad-\sum_{i, j, k} h_{i j k} \nabla_{e_{3}}\left(h_{i j 3 k}+2 \sum_{m} h_{m j} R_{m i k 3}\right) \\
& =\sum_{i, j, k} h_{i j k}\left(h_{3 i j 3 k}-h_{3 i j k 3}+\sum_{m} h_{m i k} R_{m 33 j}+3 \sum_{m} h_{3 m k} R_{m i 3 j}\right) \\
& \quad+\sum_{i, j, k, m} h_{i j k} h_{m i} \nabla_{e_{k}}\left(h_{m 3} h_{3 j}-h_{m j} h_{33}\right) \\
& \quad+\sum_{i, j, k, m} h_{i j k} h_{3 m} \nabla_{e_{k}}\left(h_{m 3} h_{i j}-h_{m j} h_{i 3}\right) \\
& \quad-2 \sum_{i, j, k, m} h_{i j k} h_{m j} \nabla_{e_{3}}\left(h_{m k} h_{i 3}-h_{m 3} h_{i k}\right)
\end{aligned}
$$




$$
\begin{aligned}
& =\sum_{i, j, k, m} h_{i j k}\left(2 h_{m i j} R_{m 33 k}+5 h_{3 m j} R_{m i 3 k}\right) \\
& +\sum_{i, j, k, m} h_{i j k} h_{m i}\left(h_{m 3 k} h_{3 j}+h_{m 3} h_{3 j k}-h_{m j k} h_{33}\right) \\
& +\sum_{i, j, k, m} h_{i j k} h_{3 m}\left(h_{m 3} h_{i j k}-h_{m j k} h_{i 3}-h_{m j} h_{i 3 k}\right) \\
& -2 \sum_{i, j, k, m} h_{i j k} h_{m j}\left(h_{m k 3} h_{i 3}-h_{m 3} h_{i k 3}\right) \\
& =\sum_{i, j, k, m} h_{i j k}\left(1+\lambda_{3} \lambda_{k}\right)\left[2 h_{m i j}\left(\delta_{m 3} \delta_{3 k}-\delta_{m k}\right)+5 h_{3 m j}\left(\delta_{m 3} \delta_{i k}-\delta_{m k} \delta_{i 3}\right)\right] \\
& +\sum_{i, k} \lambda_{3} \lambda_{i} h_{i k 3}^{2}+\sum_{i, k} \lambda_{3}^{2} h_{i k 3}^{2}-\sum_{i, j, k} \lambda_{3} \lambda_{i} h_{i j k}^{2} \\
& +\sum_{i, j, k} \lambda_{3}^{2} h_{i j k}^{2}-\sum_{j, k} \lambda_{3}^{2} h_{j k 3}^{2}-\sum_{i, k} \lambda_{3}^{2} h_{i k 3}^{2}-2 \sum_{j, k} \lambda_{3} \lambda_{j} h_{j k 3}^{2}+2 \sum_{j, k} \lambda_{3}^{2} h_{j k 3}^{2} \\
& =2 \sum_{i, j}\left(1+\lambda_{3}^{2}\right) h_{i j 3}^{2}-2 \sum_{i, j, k}\left(1+\lambda_{3} \lambda_{k}\right) h_{i j k}^{2}-5 \sum_{j, k}\left(1+\lambda_{3} \lambda_{k}\right) h_{j k 3}^{2} \\
& -\sum_{i, k} \lambda_{3} \lambda_{i} h_{i k 3}^{2}+\sum_{i, k} \lambda_{3}^{2} h_{i k 3}^{2}-\sum_{i, j, k} \lambda_{3} \lambda_{i} h_{i j k}^{2}+\sum_{i, j, k} \lambda_{3}^{2} h_{i j k}^{2} \\
& =\sum_{i, j}\left(\lambda_{3}^{2}-6 \lambda_{3} \lambda_{i}-3\right) h_{i j 3}^{2}+\sum_{i, j, k}\left(\lambda_{3}^{2}-3 \lambda_{3} \lambda_{i}-2\right) h_{i j k}^{2} \\
& =\sum_{i, j}\left[12 \bar{\lambda}^{2}-\frac{1}{3} H^{2}-3-2(H-6 \bar{\lambda}) \bar{\lambda}_{i}\right] h_{i j 3}^{2} \\
& +\sum_{i, j, k}\left[4 \bar{\lambda}^{2}-\frac{2}{9} H^{2}+\frac{2}{3} H \bar{\lambda}-2-(H-6 \bar{\lambda}) \bar{\lambda}_{i}\right] h_{i j k}^{2} \\
& =\left[2 \bar{S}-3-\frac{H^{2}}{3}-2(H-6 \bar{\lambda}) \bar{\lambda}\right](2 a) \\
& +\left(\frac{2}{3} \bar{S}-\frac{2 H^{2}}{9}+\frac{2 H \bar{\lambda}}{3}-2\right)(6 a+4 b)-(H-6 \bar{\lambda})(4 b \bar{\lambda}) \\
& =a\left(12 \bar{S}-18-2 H^{2}\right)+b\left(\frac{20}{3} \bar{S}-8-\frac{8}{9} H^{2}-\frac{4}{3} H \bar{\lambda}\right)
\end{aligned}
$$

It follows that

$$
\begin{aligned}
\frac{1}{4} \sum_{\alpha, \beta, \gamma, \sigma} h_{\alpha \beta \gamma \sigma}^{2}-\frac{4 a}{\bar{\lambda}} h_{1111}=\frac{1}{3 \bar{S}}( & \left.51 a^{2}-8 a b-36 b^{2}\right) \\
& -\left(\frac{15}{2} \bar{S}-\frac{9}{2}-\frac{1}{2} H^{2}-\frac{27}{4} H \bar{\lambda}\right) a
\end{aligned}
$$




$$
-\left(\frac{37}{6} \bar{S}+1+\frac{1}{9} H^{2}+\frac{49}{6} H \bar{\lambda}\right) b
$$

We are now in the position to conclude that $R \geq 0$ as follows: On the one hand, note that

$$
\begin{aligned}
\bar{S}-\frac{2}{3} H^{2} & =6-R \\
\frac{9}{8} \bar{S}-3 & =\bar{S}-3+\frac{3}{4} \bar{\lambda}^{2} \\
& \geq \bar{S}-3-\frac{1}{3} H^{2}+H \bar{\lambda} \\
& =\frac{1}{\bar{S}}(6 a+4 b),
\end{aligned}
$$

we have

$$
\begin{aligned}
\frac{15}{2} \bar{S}-\frac{9}{2}-\frac{1}{2} H^{2}-\frac{27}{4} H \bar{\lambda} & \geq \frac{15}{2} \bar{S}-\frac{9}{2}-\frac{1}{2} H^{2}-\frac{9}{8} H^{2}-\frac{81}{8} \bar{\lambda}^{2} \\
& =3\left(\frac{9}{8} \bar{S}-\frac{3}{2}\right)+\frac{39}{16}\left(\bar{S}-\frac{2}{3} H^{2}\right) \\
& >\frac{3}{\bar{S}}(6 a+4 b)+\frac{39}{16}(6-R) \\
\frac{37}{6} \bar{S}+1+\frac{1}{9} H^{2}+\frac{49}{6} H \bar{\lambda} & \geq \frac{37}{6} \bar{S}+1+\frac{1}{9} H^{2}-\frac{49}{36} H^{2}-\frac{49}{4} \bar{\lambda}^{2} \\
& =\frac{9}{4} \bar{S}+1+\frac{15}{8}\left(\bar{S}-\frac{2}{3} H^{2}\right) \\
& >\frac{15}{8}(6-R)
\end{aligned}
$$

It follows that the right hand side of (4.8)

$$
\begin{aligned}
R H S \leq & \frac{1}{3 \bar{S}}\left(51 a^{2}-8 a b-36 b^{2}\right)-\frac{3 a}{\bar{S}}(6 a+4 b) \\
& -\frac{39 a}{16}(6-R)-\frac{15 b}{8}(6-R) \\
= & -\frac{1}{3 \bar{S}}\left(3 a^{2}+44 a b+36 b^{2}\right)-\left(\frac{39}{16} a+\frac{15}{8} b\right)(6-R)
\end{aligned}
$$

On the other hand, by using Lemma 4.1 , we compute the left hand side of (4.8)

$$
L H S=\frac{1}{4} \sum_{\alpha, \beta, \gamma, \sigma} h_{\alpha \beta \gamma \sigma}^{2}-\frac{4 a}{\bar{\lambda}} h_{1111}
$$




$$
\begin{aligned}
& =\frac{1}{4}\left(h_{1111}^{2}+4 h_{1112}^{2}+6 h_{2211}^{2}+4 h_{2221}^{2}+h_{2222}^{2}\right)-\frac{4 a}{\bar{\lambda}} h_{1111} \\
& =\frac{1}{2} h_{1111}^{2}+2 h_{1112}^{2}+\frac{3}{2}\left(-h_{1111}-h_{3311}\right)^{2}-\frac{4 a}{\bar{\lambda}} h_{1111} \\
& =2\left(h_{1111}^{2}+h_{1112}^{2}\right)+3 h_{1111} h_{3311}+\frac{3}{2} h_{3311}^{2}-\frac{4 a}{\bar{\lambda}} h_{1111} \\
& =2\left(h_{1111}^{2}+h_{1112}^{2}\right)-\frac{2}{\bar{\lambda}}(a-b) h_{1111}+\frac{2}{3 \bar{\lambda}^{2}}(a+b)^{2} \\
& \geq-\frac{(a-b)^{2}}{2 \bar{\lambda}^{2}}+\frac{2}{3 \bar{\lambda}^{2}}(a+b)^{2} \\
& =\frac{1}{\bar{S}}\left(a^{2}+14 a b+b^{2}\right)
\end{aligned}
$$

Therefore, (4.8) yields

$$
\left(\frac{39}{16} a+\frac{15}{8} b\right)(6-R) \leq-\frac{1}{3 \bar{S}}\left(6 a^{2}+86 a b+39 b^{2}\right) \leq 0
$$

It follows that that either $R>6$ or $a=b=0$. In any case, by virtue of (2.10), we always have $R \geq 0$ as desired. This settles the case of $G(p)=0$ and thus completes the proof of the Main Theorem.

\section{REFERENCES}

[B] Bryant, R., Private conversation.

[C] Cartan, E., Sur des familles remarquables d'hypersurfaces isoparametriques dans les espaces spheriques, Math. Z. 45 (1939), 335-367

[CDK] Chern, S. S., do Carmo, M., Kobayashi, S., Minimal submanifolds of a sphere with second fundamental form of constant length, Functional analysis and related fields, 59-75, Berlin, Heidelberg, New York, Springer 1970

[Ch] Chang, S., On minimal hypersurfaces of constant scalar curvatures in $\mathbb{S}^{4}$, to appear in J. Differential Geom. 37 (1993).

[CY] Cheng, S. Y. and Yau, S. T., Hypersurfaces with constant scalar curvature, MathÄnn. 225 (1977), 195-204.

[DB] De Almeida, S. and Brito, F., Closed 3-dimensional hypersurfaces with constant mean curvature and constant scalar curvature, Duke Math. J. 61 (1990), 195206.

[L] Lawson, H. B., Local rigidity theorems for minimal hypersurfaces, Ann. Math. 89 (1969), 187-191.

[M] Munzner, H., Isoparametrische Hyperflachen in Spharen I and II, Math. Ann. 251 (1980), 57-71 and 256 (1981), 215-232.

[PT1] Peng, C. K. and Terng, C. L., Minimal hypersurface of spheres with constant scalar curvature, Ann. Math. Study 103 (1983), 177-198. 
[PT2] Peng, C. K. and Terng, C. L., The scalar curvature of minimal hypersurfaces in spheres, Math. Ann. 266 (1983), 105-113.

[S] Simons, J., Minimal varieties in a Riemannian manifold, Ann. Math. 88 (1968), 62-105.

[Y] Yau, S. T., Problem section. Ann. Math. Study 102 (1982), 693.

University of California, Los Angeles, U. S. A.

RECEIVED MAY 7, 1992. 